\title{
Diet patterns and the risk of renal cell carcinoma
}

\author{
Kiren Handa ${ }^{1}$ and Nancy Kreiger ${ }^{2,3, *}$ \\ ${ }^{1}$ Institute for Clinical Evaluative Sciences, Toronto, Ontario, Canada: ${ }^{2}$ Division of Preventive Oncology, Cancer Care \\ Ontario, 620 University Avenue, Toronto, Ontario, Canada M5G 2L7: ${ }^{3}$ Department of Public Health Sciences, \\ University of Toronto, Toronto, Ontario
}

Submitted 11 January 2001: Accepted 26 March 2002

\begin{abstract}
Objectives: Our objective was to identify food intake patterns that might be associated with the risk of renal cell carcinoma.

Design: A total of 461 cases (210 females, 251 males) were age frequency matched to population controls. Diet factors were created using factor analysis of 69 food items from a food-frequency questionnaire. These factors were modelled using logistic regression to identify those associated with renal cell carcinoma.

Setting: We investigated the role of diet in the aetiology of renal cell carcinoma using a population-based case-control study conducted in Ontario between 1995 and 1996. Subjects: Cases were Ontario residents 20 to 74 years of age identified through review of pathology reports in the Ontario Cancer Registry.

Results: A 'dessert' diet factor was positively associated with disease for both sexes (odds ratio estimate (OR) for males $=3.7,95 \%$ confidence interval (CI) 2.0-6.9; OR for females $=1.4,95 \%$ CI $0.8-2.2$, for the highest vs. lowest quartile). In males, a 'beef' diet factor was identified and was associated with an increased risk of renal cell carcinoma. Furthermore, a 'juices' diet factor also showed an association with increased risk in males (OR $=1.8,95 \%$ CI 1.0-3.1). For females, a positive association was observed between renal cell carcinoma and an 'unhealthy' diet factor $(\mathrm{OR}=1.4$, 95\% CI 0.8-2.4).

Conclusions: Our findings confirmed that high-fat and high-protein diets might be risk factors for renal cell carcinoma. The data also suggest an increased risk associated with juice intake, a finding not previously reported.
\end{abstract}

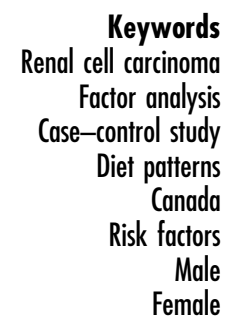

The role of diet in the aetiology of renal cell carcinoma is unresolved. The most consistent results show increased risks associated with the consumption of foods such as meat, eggs and dairy products and reduced risk for fruits and vegetables ${ }^{1-7}$. Studies have shown a higher risk associated with protein and fat ${ }^{8,9}$ and potential decreased risks associated with vitamin $\mathrm{C}$ and vitamin $\mathrm{E}^{8-13}$. These studies have assessed diet as specific foods or nutrients. Yet individuals consume combinations of nutrients or foods at a time, and therefore are likely to experience effects that are a consequence of the interactions among these dietary items. Disease associations with diet may not be captured entirely by examining individual constituents.

The objective of this study was to identify dietary patterns associated with renal cell carcinoma, by reducing a large number of food items to a more parsimonious number of factors based on the correlations among foods.

\section{Subjects and methods}

Data came from the Ontario portion of the Enhanced Cancer Surveillance (ECS) of the Laboratory Centre for
Disease Control at Health Canada, a population-based multi-cancer case-control study ${ }^{14}$. Analyses were restricted to Ontario data because the dietary component of the questionnaire was different from that in other provinces. Cases were Ontario residents 20 to 74 years of age, with a histologically confirmed cancer diagnosed between January 1995 and December 1996, identified through review of pathology reports in the Ontario Cancer Registry ${ }^{15}$.

Controls were 20 to 74 years of age, Ontario residents, identified through the population-based assessment rolls of the Ontario Ministry of Finance, and selected to yield a control sample with a sex and 5-year age distribution similar to all cases combined.

Physicians were asked for consent to contact cases, to confirm the cancer diagnosis and to provide the patient's vital status, address, telephone number and next-of-kin (if necessary). Cases and controls were mailed an explanatory letter and a self-administered questionnaire. Followup to non-respondents included postcard, letter and telephone. Questionnaires were reviewed for comprehensibility and completeness, and subjects were telephoned to supply missing information or clarification. 
In total, 842 kidney cancer cases were identified ( 462 males and 380 females). Of those, 652 were mailed questionnaires while the remainder were not eligible by age $(n=51)$, deceased $(n=31)$, physician refusal/late consent $(n=60)$ or unknown physician/subject address $(n=48)$. Five hundred and thirty-five kidney cancer cases returned questionnaires $(82.1 \%)$. Of these, nine did not meet diagnostic criteria and 16 were proxy respondents, and were excluded.

Questionnaires were sent to 2941 potential controls (1406 males, 1535 females); 1929 (901 males, 1028 females) were returned (64.1\% response males, $67.0 \%$ females). Of these, 1588 were within the overall age range for renal cell carcinoma cases (787 males, 801 females). From these, male and female controls were frequency matched to the cases based on 5-year age groups, 1:1 for males and 2:1 for females.

Frequency of intake of 69 food items was entered as one of nine categories ranging from 'never or less than 1 per month' to ' 6 or more times per day' in the diet questionnaire using Canadian nutrient data ${ }^{16}$. The median frequencies of food use reported on a monthly or daily basis were converted to a period of weekly food use. For many items, where more than daily intake was rare, the data were collapsed to a maximum of $\geq 7$ times per week.

To avoid extensive omission of cases and controls due to missing values, responses were imputed for subjects missing $\leq 5 \%$ of food frequency variables. Imputed values were randomly selected from the intake frequencies provided by the subset of cases and controls with complete diet information within each case/control, sex, age and smoking stratum. Approximately $90 \%$ of cases and controls had $\leq 5 \%$ of diet variables missing. The percentage of cases and controls with imputed diet variables did not differ. Analyses were based on 461 cases (210 females, 251 males) and 672 controls ( 422 females, 250 males).

Factor analysis ${ }^{17}$ was used to identify dietary factors, or combinations of foods, consumed in the study sample. Male and female controls were used to generate two separate sets of diet patterns. A correlation matrix of the original 69 dietary variables was created, and maximum likelihood factor analysis followed by varimax rotation was used to create a parsimonious set of factors. Only factors with eigenvalues $\geq 1.5$ were selected. Dietary factors consisted of those items in each factor that correlated with the factor with factor loading of $\leq-0.20$ or $\geq 0.30$. Factor scores for each dietary factor were calculated for cases and controls by multiplying the standardised scoring coefficient of each food included in the factor by the value reported in the food-frequency questionnaire, and summing across all foods in the factor. Subjects were assigned scores to indicate the degree to which their diet adhered to each of the factors. Labels were assigned to each factor based on an approximate description of the food items that were most highly represented.
Sex-specific unconditional logistic regression methods were used ${ }^{18}$. Odds ratios (ORs) and 95\% confidence intervals (CIs) were estimated using EGRET ${ }^{19}$. Stepwise regression analysis was used to determine which diet patterns would be included in the final logistic regression model. Factors with a $P$-value $\leq 0.20$ were included in the stepwise model. Evaluation of the effects of the dietary patterns was carried out while fitting the final logistic regression model. Factors that were significantly associated with an increased risk of renal cell carcinoma were included ( $P$-value $\leq 0.05$ ). Likelihood ratio statistics were used to assess the contribution of the individual variables in a model and were calculated based on the reduction in residual deviance after the addition of the variable to a prior fitted model. Potential confounding variables were age, smoking status (never/former/current) and body mass index (BMI), and were included in the modelling.

\section{Results}

Tables 1 and 2 present dietary factors from the factor analysis of the female and male controls, respectively. For females, diet variables converged into eight factors that explained $15.5 \%$ of the total variance. Thirteen dietary factors explaining $24 \%$ of the variance were generated for males. Of these, the first eight explained most of this (17.5\%).

Although the numerical values of the factor loading were different for males and females, Factor I for both was highly correlated with many fruits and vegetables. Factor II consisted of 'dessert' items. Similarly, a 'meat' diet factor was identified for both sexes (Factor IV in females, Factor VIII in males). Another dietary factor consisting of meat was identified in males in Factor VII, in which beef was the only food variable represented.

In males, a 'fruit juice' dietary factor was represented in Factor $\mathrm{V}$ : frozen, powdered and fresh juices. In females, Factor VII included fruit juices that were consumed often, while the intake of liquor and wine was infrequent. Factor VIII in females was positively associated with white bread but inversely associated with broccoli, skimmed milk, $1 \%$ milk and dark bread, which might be termed an 'unhealthy diet'.

Tables 3 and 4 present the distribution of age, previously identified risk factors and the diet patterns for females and males, respectively. Variables that were associated with increased risk in females included BMI and smoking status. The highest quartile for BMI was associated with a 2.4-fold increased risk (95\% CI 1.53.8). Current and former smokers were at somewhat increased risk, although this relationship was significant only among former smokers (OR $=1.7,95 \%$ CI 1.1-2.5). While increasing pack-years were associated with increased risk, all confidence intervals included unity.

For females, Factor I ('fruits and vegetables') was associated with reduced renal cell carcinoma risk, with the 
Table 1 Dietary food patterns identified for female controls $(n=422)$

Factor loading

\begin{tabular}{|c|c|c|c|c|c|c|c|c|}
\hline \multirow[b]{2}{*}{ Food/beverage } & \multicolumn{8}{|c|}{ Factor loading } \\
\hline & $\begin{array}{c}\text { Factor I } \\
\text { Fruits/vegetables }\end{array}$ & $\begin{array}{l}\text { Factor II } \\
\text { Dessert }\end{array}$ & $\begin{array}{c}\text { Factor III } \\
\text { Miscellaneous + }\end{array}$ & $\begin{array}{c}\text { Factor IV } \\
\text { Meat }\end{array}$ & $\begin{array}{c}\text { Factor V } \\
\text { Miscellaneous - }\end{array}$ & $\begin{array}{l}\text { Factor } \mathrm{VI} \\
\text { High protein }\end{array}$ & $\begin{array}{l}\text { Factor VII } \\
\text { Beverage }\end{array}$ & $\begin{array}{l}\text { Factor VIII } \\
\text { Unhealthy }\end{array}$ \\
\hline Coffee & & & & & 0.32 & & & \\
\hline Powdered drinks & & & & & & & & \\
\hline Bottled water & & & 0.43 & & & & & \\
\hline $1 \%$ milk & & & & & & & & -0.24 \\
\hline Skimmed milk & & & & & & & & -0.23 \\
\hline \multicolumn{9}{|l|}{ Fresh citrus juice } \\
\hline Other fresh juice & & & & & & & 0.36 & \\
\hline Tomato/vegetable juice & & & & & 0.41 & & & \\
\hline Frozen juice/drink & & & & & & & 0.46 & \\
\hline \multicolumn{9}{|l|}{ Beer } \\
\hline Wine & & & & & & & -0.20 & -0.20 \\
\hline Liquor & & & & & & & -0.20 & \\
\hline Apples/pears & 0.39 & & & & -0.23 & & & \\
\hline Oranges & 0.37 & & & & & & & \\
\hline Bananas & 0.32 & & & & & & 0.45 & \\
\hline Cantaloupe & & & 0.42 & & & & & \\
\hline Other fruit - fresh, canned & 0.44 & & & & & & & \\
\hline Tomatoes & 0.37 & & 0.34 & & & & & \\
\hline Carrots & 0.54 & & & & & & & \\
\hline Broccoli & 0.39 & & & & & & & -0.21 \\
\hline Cabbage* $^{\star}$ & 0.41 & & & & & & & \\
\hline Spinach or other greens & 0.49 & & & & & & & \\
\hline Yellow squash & & & 0.37 & & & & & \\
\hline Other vegetables & 0.59 & & & & & & & \\
\hline \multicolumn{9}{|l|}{ Soups with vegetables } \\
\hline Potatoes & 0.39 & & -0.20 & & 0.32 & & & \\
\hline French fries & & 0.37 & & & & & & \\
\hline \multirow{2}{*}{\multicolumn{9}{|c|}{ Baked beans or lentils }} \\
\hline & & & & & & & & \\
\hline $\begin{array}{l}\text { Bran or granola cereals } \\
\text { Cooked cereals }\end{array}$ & 0.35 & & & & & & & \\
\hline White bread & & & & & & & & 0.37 \\
\hline Dark bread & & & & & & & & -0.28 \\
\hline Rice & & & & & -0.40 & 0.60 & & \\
\hline Macaroni, spaghetti & & & 0.33 & & & & & \\
\hline Chicken or turkey & & & & & & 0.32 & & \\
\hline \multirow{2}{*}{\multicolumn{9}{|c|}{$\begin{array}{l}\text { Beef/pork/lamb - main dish } \\
\text { Beef/pork/lamb - mixed dish }\end{array}$}} \\
\hline & \multicolumn{8}{|c|}{ Beef/pork/lamb - mixed dish } \\
\hline Hamburger & & & & 0.48 & & & & \\
\hline Hot dogs & & & & 0.64 & & & & \\
\hline Luncheon meats & & & & 0.46 & & & & \\
\hline \multicolumn{9}{|l|}{ Smoked meat, corned beef } \\
\hline \multirow{2}{*}{\multicolumn{9}{|c|}{$\begin{array}{l}\text { Bacon } \\
\text { Sausage }\end{array}$}} \\
\hline Sausage & & & & & & & & \\
\hline Liver & & 0.47 & & & & & & \\
\hline Fish - fresh, frozen, canned & 0.30 & & & & & & & \\
\hline Fish - smoked, salted, dried & & & 0.43 & & & & & \\
\hline Eggs & & & & & & 0.36 & & \\
\hline \multicolumn{9}{|l|}{ Cheese other than cottage } \\
\hline Cake & & 0.51 & & & & & & \\
\hline Cookies & & 0.44 & & & & & & \\
\hline Doughnuts & & 0.61 & & & & & & \\
\hline Pies & & 0.37 & & & & & & \\
\hline Ice cream & & 0.46 & & & & & & \\
\hline Chocolate & & 0.55 & & & & & & \\
\hline Potato chips & & 0.30 & & 0.35 & & & & \\
\hline Peanut butter & & & & & & & & -0.27 \\
\hline \multicolumn{9}{|l|}{ Nuts } \\
\hline Butter on bread & & & & & 0.32 & & & \\
\hline Margarine on bread & & & -0.20 & & & & & \\
\hline Mayonnaise/salad dressing & & & & & 0.40 & & & \\
\hline Percentage of variance & 3.13 & 2.45 & 1.85 & 1.78 & 1.70 & 1.67 & 1.64 & 1.28 \\
\hline
\end{tabular}

Food items with factor loadings $\leq-0.20$ and $\geq 0.30$ were included.

${ }^{*}$ Cabbage includes cauliflower and Brussels sprouts. 


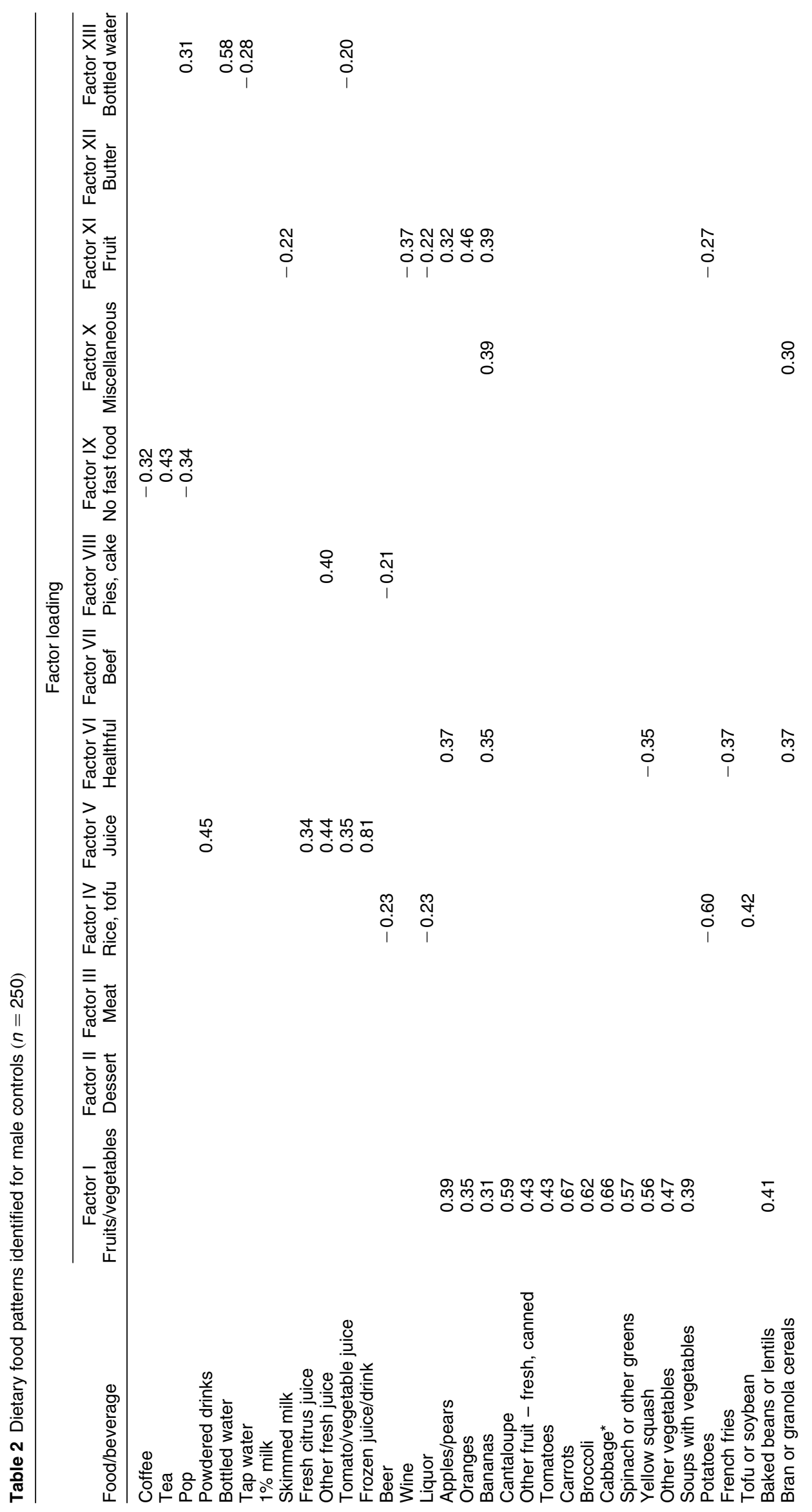




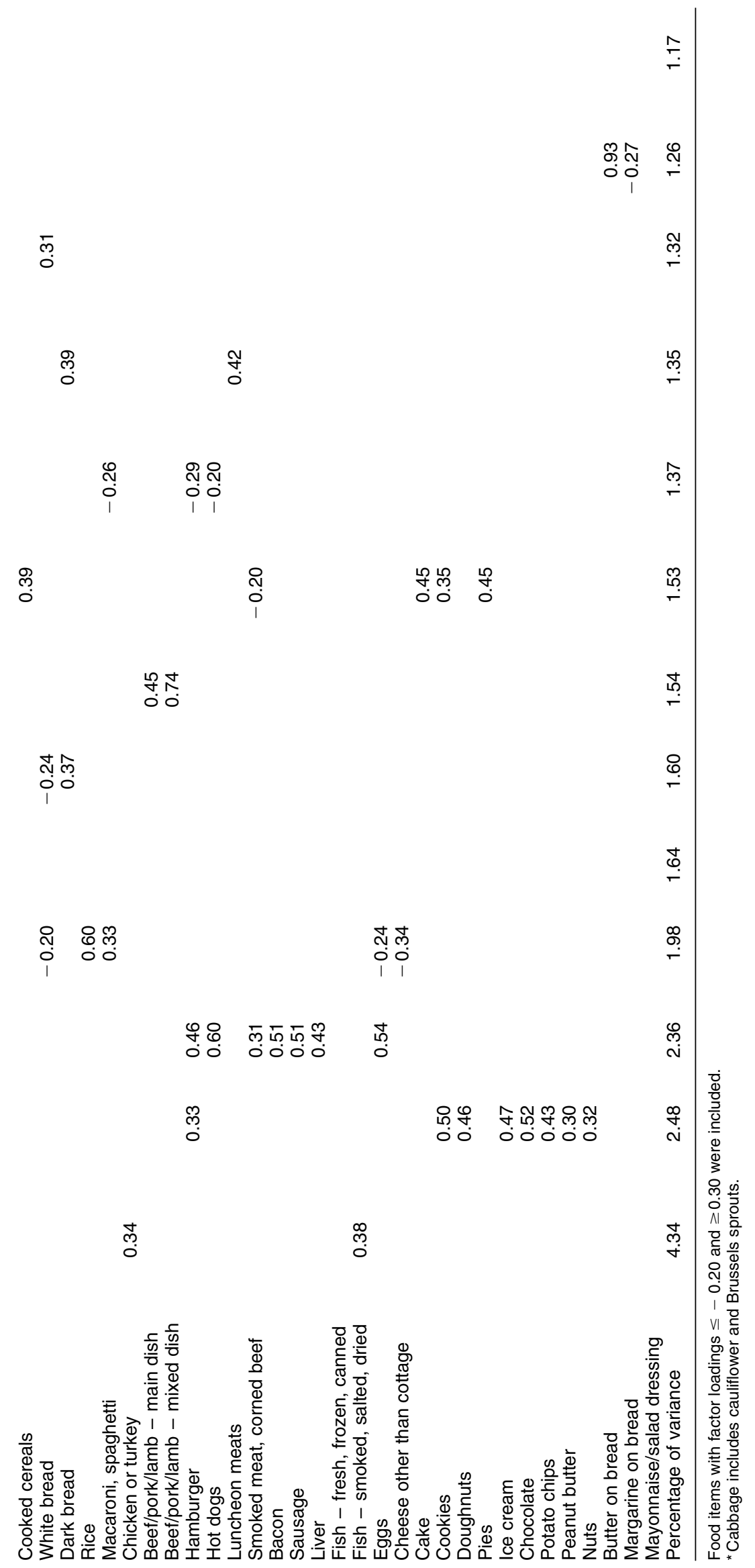


Table 3 Age-adjusted ORs and 95\% Cls for renal cell carcinoma and risk factors for age-matched females

\begin{tabular}{|c|c|c|c|}
\hline & Cases, $n(\%)$ & Controls, $n(\%)$ & OR $(95 \% \mathrm{Cl})$ \\
\hline \multicolumn{4}{|l|}{ Age group (years) } \\
\hline $35-39$ & $10(4.8)$ & $19(4.5)$ & \\
\hline $40-44$ & $17(8.1)$ & $34(8.1)$ & \\
\hline $45-49$ & $25(11.9)$ & $48(11.4)$ & \\
\hline $50-54$ & $31(14.8)$ & $63(14.9)$ & \\
\hline $55-59$ & $25(11.9)$ & $51(12.1)$ & \\
\hline $60-64$ & $36(17.1)$ & 79 (18.7) & \\
\hline $65-69$ & $32(15.2)$ & $62(14.7)$ & \\
\hline $70-74$ & $34(16.2)$ & $66(15.6)$ & \\
\hline \multicolumn{4}{|l|}{$\mathrm{BMI}^{*}\left(\mathrm{~kg} \mathrm{~m}^{-2}\right)$} \\
\hline$\leq 22.10$ & $36(17.1)$ & $108(25.6)$ & 1.0 \\
\hline $22.11-24.61$ & 39 (18.6) & $107(25.4)$ & $1.1(0.7-1.9)$ \\
\hline $24.62-27.93$ & $53(25.2)$ & $100(23.7)$ & $1.6(1.0-2.7)$ \\
\hline$>27.93$ & $81(38.6)$ & $104(24.6)$ & $2.4(1.5-3.8)$ \\
\hline \multicolumn{4}{|l|}{ Education } \\
\hline$<11$ years post secondary & $92(43.8)$ & $151(35.8)$ & 1.0 \\
\hline$\geq 12$ years post secondary & 39 (18.6) & $86(20.4)$ & $0.7(0.5-1.2)$ \\
\hline$<4$ years college & $61(29.0)$ & $137(32.5)$ & $0.7(0.5-1.1)$ \\
\hline$\geq 4$ years college & $18(8.6)$ & $48(11.4)$ & $0.6(0.3-1.1)$ \\
\hline \multicolumn{4}{|l|}{ Smoking status } \\
\hline Never & $99(47.1)$ & $239(56.6)$ & 1.0 \\
\hline Former & $61(29.0)$ & $88(20.9)$ & $1.7(1.1-2.5)$ \\
\hline Current & $50(23.8)$ & $94(22.3)$ & $1.3(0.8-1.9)$ \\
\hline \multicolumn{4}{|l|}{ Pack-yearst } \\
\hline Never smokers & $99(47.1)$ & $239(56.6)$ & 1.0 \\
\hline Former QI & $26(12.4)$ & $40(9.5)$ & $1.6(0.9-2.7)$ \\
\hline Former QII & $16(7.6)$ & $16(3.8)$ & $2.4(1.2-5.0)$ \\
\hline Former QIII & $11(5.2)$ & $20(4.7)$ & $1.3(0.6-2.9)$ \\
\hline Former QIV & $8(3.8)$ & $12(2.8)$ & $1.6(0.6-4.1)$ \\
\hline Never smokers & 99 (47.1) & $239(56.6)$ & 1.0 \\
\hline Current QI & $2(1.0)$ & $5(1.2)$ & $1.0(0.2-5.0)$ \\
\hline Current QII & $10(4.8)$ & $30(7.1)$ & $0.8(0.4-1.7)$ \\
\hline Current QIII & $18(8.6)$ & $25(5.9)$ & $1.7(0.9-3.3)$ \\
\hline Current QIV & $20(9.5)$ & $34(8.1)$ & $1.4(0.8-2.6)$ \\
\hline \multicolumn{4}{|c|}{ Smoking cessation (former smokers only) } \\
\hline$\geq 10$ years & $40(65.6)$ & $60(68.2)$ & 1.0 \\
\hline$<10$ years & $21(34.4)$ & $28(31.8)$ & $1.1(0.6-2.3)$ \\
\hline \multicolumn{4}{|c|}{ Smoke age (former and current smokers) } \\
\hline$<20$ years of age & $31(27.9)$ & $65(35.7)$ & 1.0 \\
\hline$\geq 20$ years of age & 79 (71.2) & $118(64.8)$ & $1.4(0.8-2.3)$ \\
\hline \multicolumn{4}{|l|}{ Diet factor } \\
\hline \multicolumn{4}{|l|}{ Factor I - Fruits and vegetables } \\
\hline QI & $62(29.5)$ & $105(24.9)$ & 1.0 \\
\hline QII & $54(25.7)$ & $106(25.1)$ & $0.9(0.5-1.3)$ \\
\hline QIII & $62(29.5)$ & $106(25.1)$ & $1.0(0.6-1.5)$ \\
\hline QIV & $32(15.2)$ & $105(24.9)$ & $0.5(0.3-0.8)$ \\
\hline \multicolumn{4}{|l|}{ Factor II - Desserts } \\
\hline QI & $47(22.4)$ & $105(24.9)$ & 1.0 \\
\hline QII & $33(15.7)$ & $106(25.1)$ & $0.7(0.4-1.2)$ \\
\hline QIII & $62(29.5)$ & $105(24.9)$ & $1.3(0.8-2.1)$ \\
\hline QIV & $68(32.4)$ & $106(25.1)$ & $1.4(0.9-2.3)$ \\
\hline \multicolumn{4}{|l|}{ Factor III - Miscellaneous+ } \\
\hline QI & $66(31.4)$ & $105(24.9)$ & 1.0 \\
\hline QII & $58(27.6)$ & $106(25.1)$ & $0.9(0.5-1.4)$ \\
\hline QIII & $41(19.5)$ & $105(24.9)$ & $0.6(0.4-1.0)$ \\
\hline QIV & $45(21.4)$ & $106(25.1)$ & $0.7(0.4-1.1)$ \\
\hline Factor IV - Meat & & & \\
\hline QI & $40(19.0)$ & $105(24.9)$ & 1.0 \\
\hline QII & $53(25.2)$ & $106(25.1)$ & $1.3(0.8-2.2)$ \\
\hline QIII & $49(23.3)$ & $106(25.1)$ & $1.2(0.7-2.0)$ \\
\hline QIV & $68(32.4)$ & $105(24.9)$ & $1.7(1.1-2.8)$ \\
\hline Factor V - Miscellaneous - & & & \\
\hline QI & $33(15.7)$ & $106(25.1)$ & 1.0 \\
\hline QII & $51(24.3)$ & $105(24.9)$ & $1.6(0.9-2.6)$ \\
\hline QIII & 67 (31.9) & $105(24.9)$ & $2.1(1.2-3.4)$ \\
\hline QIV & $59(28.1)$ & $106(25.1)$ & $1.8(1.1-3.0)$ \\
\hline Factor VI - High protein & & & \\
\hline QI & $39(18.6)$ & $106(25.1)$ & 1.0 \\
\hline QII & $63(30.0)$ & $105(24.9)$ & $1.6(1.0-2.7)$ \\
\hline QIII & $55(26.2)$ & $106(25.1)$ & $1.4(0.9-2.3)$ \\
\hline QIV & $53(25.2)$ & $105(24.9)$ & $1.4(0.8-2.3)$ \\
\hline
\end{tabular}


Table 3. Continued

\begin{tabular}{|c|c|c|c|}
\hline & Cases, $n(\%)$ & Controls, $n(\%)$ & OR $(95 \% \mathrm{Cl})$ \\
\hline \multicolumn{4}{|c|}{ Factor VII - Beverages } \\
\hline QI & $48(22.9)$ & $106(25.1)$ & 1.0 \\
\hline QII & $55(26.2)$ & $105(24.9)$ & $1.2(0.7-1.9)$ \\
\hline QIII & $61(29.0)$ & $106(25.1)$ & $1.3(0.8-2.0)$ \\
\hline QIV & $46(21.9)$ & $105(24.9)$ & $1.0(0.6-1.6)$ \\
\hline \multicolumn{4}{|c|}{ Factor VIII - Unhealthy } \\
\hline QI & $40(19.0)$ & $106(25.1)$ & 1.0 \\
\hline QII & $46(21.9)$ & $105(24.9)$ & $1.2(0.7-1.9)$ \\
\hline QIII & 68 (32.4) & $106(25.1)$ & $1.7(1.1-2.7)$ \\
\hline QIV & $56(26.7)$ & $105(24.9)$ & $1.4(0.9-2.3)$ \\
\hline
\end{tabular}

${ }^{*} \mathrm{BMI}$ - body mass index.

† Quartiles of pack-years determined using former and current smokers combined: QI $<5.1 ; 5.1 \leq$ QII $<17.3 ; 17.3 \leq$ QIII $<31.1$; QIV $\geq 31.1$

highest consumption category associated with an OR of 0.5 (95\% CI 0.3-0.8). A reduced risk was also associated with the highest intake levels of Factor III, the 'miscellaneoust' factor (OR $=0.7$, 95\% CI 0.4-1.1). Elevated risks were observed for diet factor IV ('meat') and diet factor $\mathrm{V}$ ('miscellaneous - ').

For males (Table 4), high BMI was associated with a significant increase in risk $(\mathrm{OR}=3.5,95 \%$ CI 2.1-6.0). Reduced odds ratio estimates were observed for the most educated (OR $=0.7,95 \%$ CI 0.4-1.1). Former and current smokers in the highest quartiles had a non-significant increased risk relative to non-smokers (current smokers $\mathrm{OR}=1.3,95 \% \mathrm{CI} 0.8-2.1$; former smokers $\mathrm{OR}=1.6,95 \%$ CI 1.0-2.5). The time since smoking cessation for former smokers did not appear to affect the risk of renal cell carcinoma. Smokers starting to smoke after the age of 20 were at an elevated risk relative to smokers who started at an early age; this risk estimate, however, was not significant $(\mathrm{OR}=1.3,95 \% \mathrm{CI} 0.8-2.2)$.

A reduction in risk was observed among the highest quartile of Factor I ('fruits and vegetables') relative to the lowest (OR $=0.6,95 \%$ CI $0.4-1.0$ ). Most notable was the significant increased risk associated with the highest intake level of Factor II ('desserts') (OR = 3.9, 95\% CI 2.26.9). Increased odds ratio estimates were also observed for Factors III ('meats') and VII ('beef').

Table 5 displays the final logistic regression models. For both males and females, a 'desserts' diet pattern was associated with an increased risk of renal cell carcinoma. Further, among males, an increased risk associated with the consumption of a 'beef' diet (Factor VII) was observed. The 'juices' pattern (Factor V) was associated with an increased risk of renal cell carcinoma for males $(\mathrm{OR}=1.7$, 95\% CI 1.0-3.1). For females, a positive association between renal cell carcinoma and the 'unhealthy' factor (Factor VIII) was observed (OR $=1.4,95 \%$ CI $0.8-2.4$ ).

\section{Discussion}

Disease risk cannot be determined by the presence or absence of any single food or nutrient. Rather it is the selection of foods in certain amounts and combinations that is more likely to play a role in carcinogenesis. The majority of epidemiological studies proposing diet as a risk factor for renal cell carcinoma have examined the association of cancer risk with the intake of single nutrients, foods or food groups. Failing to control for the correlations between foods, these studies may not have adequately considered the metabolic consequences of food items consumed concurrently or the inverse relationships of food intake.

In an attempt to control for the correlations among foods, factor analysis was used to derive dietary patterns as risk factors. The results of these analyses confirm those of previous studies in which beef, high-fat and high-protein diets are associated with renal cell carcinoma. Most of the evidence linking diet with renal cell carcinoma suggests that high protein consumption, particularly from meat, eggs or milk, is positively associated with the disease $\mathrm{e}^{1,2,4,6,7,12,13,20,21}$. Recently, a study ascertained that the population-attributable risk associated with protein intake was $19 \%$ for any intake above the lowest quartile ${ }^{22}$. Our results add evidence that this type of diet may be considered an important risk factor for renal cell carcinoma.

Despite the potential association with fat, few studies have examined the risk of renal cell carcinoma associated with dessert items. Maclure and Willett ${ }^{1}$ found elevated but non-significant risks associated with moderate consumption of ice cream, pie, cake, doughnuts and cookies. Another study reported no association between a dessert food group and renal cell carcinoma ${ }^{13}$. Since fats have been associated with renal cell carcinoma ${ }^{6,8,9,12,23}$, a diet rich in desserts may be associated with risk.

Our study did not consider energy adjustment of food intake. As obesity is an established risk factor of renal cell carcinoma, it is possible that overeating is a primary cause of the disease. In this situation, nutrients contributing to calories (proteins, fats, carbohydrates and alcohol) might be considered as the primary exposures that lead to increased calorie intake, which in turn cause disease ${ }^{24}$. Therefore, adjustment for caloric intake would likely result 
Table 4 Age-adjusted ORs and 95\% Cls for renal cell carcinoma and previously identified risk factors for age-matched males

\begin{tabular}{|c|c|c|c|}
\hline & Cases, $n(\%)$ & Controls, $n(\%)$ & OR $(95 \% \mathrm{Cl})$ \\
\hline \multicolumn{4}{|l|}{ Age group (years) } \\
\hline $25-29$ & $1(0.4)$ & $1(0.4)$ & \\
\hline $30-34$ & $2(0.8)$ & $2(0.8)$ & \\
\hline $35-39$ & $4(1.6)$ & $4(1.6)$ & \\
\hline $40-44$ & $18(7.2)$ & $18(7.2)$ & \\
\hline $45-49$ & $23(9.2)$ & $24(9.6)$ & \\
\hline $50-54$ & $31(12.4)$ & $30(12.0)$ & \\
\hline $55-59$ & $43(17.1)$ & $44(17.6)$ & \\
\hline $60-64$ & $41(16.3)$ & $41(16.4)$ & \\
\hline $65-69$ & $47(18.7)$ & $44(17.6)$ & \\
\hline $70-74$ & 41 (16.3) & $42(16.8)$ & \\
\hline \multicolumn{4}{|l|}{$\mathrm{BMI}^{*}\left(\mathrm{~kg} \mathrm{~m}^{-2}\right)$} \\
\hline$\leq 23.77$ & $32(12.7)$ & $62(24.8)$ & 1.0 \\
\hline $23.78-25.71$ & 35 (13.9) & $63(25.2)$ & $1.1(0.6-1.9)$ \\
\hline $25.72-28.08$ & 68 (27.1) & $62(24.8)$ & $2.1(1.2-3.7)$ \\
\hline$>28.08$ & $115(45.8)$ & $63(25.2)$ & $3.5(2.1-6.0)$ \\
\hline \multicolumn{4}{|l|}{ Education } \\
\hline$<11$ years post secondary & $92(36.7)$ & 83 (33.2) & 1.0 \\
\hline$\geq 12$ years post secondary & $51(20.3)$ & $44(17.6)$ & $1.0(0.6-1.7)$ \\
\hline$<4$ years college & 76 (30.3) & $79(31.6)$ & $0.9(0.6-1.3)$ \\
\hline$\geq 4$ years college & $32(12.7)$ & $44(17.6)$ & $0.7(0.4-1.1)$ \\
\hline \multicolumn{4}{|l|}{ Smoking status } \\
\hline Never & $56(22.3)$ & $74(29.6)$ & 1.0 \\
\hline Former & $129(51.4)$ & 108 (43.2) & $1.6(1.0-2.5)$ \\
\hline Current & $66(26.3)$ & $68(27.2)$ & $1.3(0.8-2.1)$ \\
\hline \multicolumn{4}{|l|}{ Pack-years $†$} \\
\hline Never smokers & $56(22.3)$ & $74(29.6)$ & 1.0 \\
\hline Former QI & $43(17.1)$ & $33(13.2)$ & $1.7(1.0-3.1)$ \\
\hline Former QII & $32(12.7)$ & $30(12.0)$ & $1.4(0.8-2.6)$ \\
\hline Former QIII & $24(9.6)$ & $25(10.0)$ & $1.3(0.7-2.5)$ \\
\hline Former QIV & $27(10.8)$ & $18(7.2)$ & $2.1(1.0-4.1)$ \\
\hline Never smokers & $56(22.3)$ & $74(29.6)$ & 1.0 \\
\hline Current QI & $7(2.8)$ & $9(3.6)$ & $1.0(0.4-2.9)$ \\
\hline Current QII & $8(3.2)$ & $14(5.6)$ & $0.7(0.3-1.9)$ \\
\hline Current QIII & $24(9.6)$ & $21(8.4)$ & $1.5(0.8-3.0)$ \\
\hline Current QIV & $26(10.4)$ & $24(9.6)$ & $1.5(0.8-2.8)$ \\
\hline \multicolumn{4}{|c|}{ Smoking cessation (former smokers only) } \\
\hline$\geq 10$ years & $96(74.4)$ & 78 (72.2) & 1.0 \\
\hline$<10$ years & $33(25.6)$ & $30(27.8)$ & $0.9(0.5-1.5)$ \\
\hline \multicolumn{4}{|c|}{ Smoke age (former and current smokers) } \\
\hline$<20$ years of age & $34(17.4)$ & $38(21.6)$ & 1.0 \\
\hline$\geq 20$ years of age & $161(82.6)$ & 136 (77.3) & $1.3(0.8-2.2)$ \\
\hline \multicolumn{4}{|l|}{ Diet factor } \\
\hline \multicolumn{4}{|l|}{ Factor I - Fruits and vegetables } \\
\hline QI & 77 (30.6) & $63(25.2)$ & 1.0 \\
\hline QII & $58(23.1)$ & $62(24.8)$ & $0.8(0.5-1.2)$ \\
\hline QIII & $69(27.5)$ & $62(24.8)$ & $0.9(0.6-1.5)$ \\
\hline QIV & $47(18.7)$ & $63(25.2)$ & $0.6(0.4-1.0)$ \\
\hline \multicolumn{4}{|l|}{ Factor II - Desserts } \\
\hline QI & $24(9.6)$ & $63(25.2)$ & 1.0 \\
\hline QII & $57(22.7)$ & $62(24.8)$ & $2.4(1.3-4.4)$ \\
\hline QIII & 79 (31.5) & $63(25.2)$ & $3.3(1.9-5.9)$ \\
\hline QIV & 91 (36.2) & $62(24.8)$ & $3.9(2.2-6.9)$ \\
\hline \multicolumn{4}{|l|}{ Factor III - Meat } \\
\hline QI & $39(15.5)$ & $62(24.8)$ & 1.0 \\
\hline QII & 67 (26.7) & $63(25.2)$ & $1.7(1.0-2.9)$ \\
\hline QIII & 77 (30.7) & $62(24.8)$ & $2.0(1.2-3.3)$ \\
\hline QIV & $68(27.1)$ & $63(25.2)$ & $1.7(1.0-2.9)$ \\
\hline \multicolumn{4}{|l|}{ Factor IV - Rice, tofu } \\
\hline QI & $63(25.1)$ & $62(24.8)$ & 1.0 \\
\hline QII & $62(24.7)$ & $63(25.2)$ & $1.0(0.6-1.6)$ \\
\hline QIII & 79 (31.5) & $63(25.2)$ & $1.2(0.8-2.0)$ \\
\hline QIV & 47 (18.7) & $62(24.8)$ & $0.7(0.4-1.3)$ \\
\hline \multicolumn{4}{|l|}{ Factor V - Juices } \\
\hline QI & $43(17.1)$ & $62(24.8)$ & 1.0 \\
\hline QII & $40(15.9)$ & $63(25.2)$ & $0.9(0.5-1.6)$ \\
\hline QIII & $95(37.8)$ & $62(24.8)$ & $2.2(1.3-3.7)$ \\
\hline QIV & $73(29.1)$ & $63(25.2)$ & $1.7(1.0-2.9)$ \\
\hline
\end{tabular}


Table 4. Continued

\begin{tabular}{|c|c|c|c|}
\hline & Cases, $n(\%)$ & Controls, $n(\%)$ & OR $(95 \% \mathrm{Cl})$ \\
\hline \multicolumn{4}{|c|}{ Factor VI - Fruit, dark bread } \\
\hline QI & $51(20.3)$ & $62(24.8)$ & 1.0 \\
\hline QII & $80(31.9)$ & $63(25.2)$ & $1.5(0.9-2.5)$ \\
\hline QIII & $71(28.3)$ & $63(25.2)$ & $1.4(0.8-2.3)$ \\
\hline QIV & 49 (19.5) & $62(24.8)$ & $1.0(0.6-1.6)$ \\
\hline \multicolumn{4}{|c|}{ Factor VII - Beef } \\
\hline QI & 49 (19.5) & $62(24.8)$ & 1.0 \\
\hline QII & $51(20.3)$ & $63(25.2)$ & $1.0(0.6-1.7)$ \\
\hline QIII & $91(36.3)$ & $62(24.8)$ & $1.9(1.1-3.0)$ \\
\hline QIV & $60(23.9)$ & $63(25.2)$ & $1.2(0.7-2.0)$ \\
\hline \multicolumn{4}{|c|}{ Factor VIII - Pies, cakeł } \\
\hline QI & $47(18.7)$ & $62(24.8)$ & 1.0 \\
\hline QII & $64(24.3)$ & $63(25.2)$ & $1.3(0.8-2.2)$ \\
\hline QIII & $66(26.3)$ & $63(25.2)$ & $1.4(0.8-2.3)$ \\
\hline QIV & $74(29.5)$ & $62(24.8)$ & $1.6(0.9-2.6)$ \\
\hline \multicolumn{4}{|c|}{ Factor IX - No fast food } \\
\hline QI & $47(18.7)$ & $62(24.8)$ & 1.0 \\
\hline QII & $68(27.1)$ & $63(25.2)$ & $1.4(0.9-2.3)$ \\
\hline QIII & $68(27.1)$ & $63(25.2)$ & $1.4(0.9-2.4)$ \\
\hline QIV & $68(27.1)$ & $62(24.8)$ & $1.5(0.9-2.5)$ \\
\hline \multicolumn{4}{|c|}{ Factor X - Miscellaneous } \\
\hline QI & $51(20.3)$ & $62(24.8)$ & 1.0 \\
\hline QII & $70(27.9)$ & $63(25.2)$ & $1.4(0.8-2.2)$ \\
\hline QIII & $74(29.5)$ & $62(24.8)$ & $1.5(0.9-2.4)$ \\
\hline QIV & $56(22.3)$ & $63(25.2)$ & $1.1(0.6-1.8)$ \\
\hline \multicolumn{4}{|c|}{ Factor XI - Fruit } \\
\hline QI & $51(20.3)$ & $63(25.2)$ & 1.0 \\
\hline QII & $68(27.1)$ & $62(24.8)$ & $1.4(0.8-2.2)$ \\
\hline QIII & $69(27.5)$ & 63 (25.2) & $1.4(0.8-2.2)$ \\
\hline QIV & $63(25.1)$ & $62(24.8)$ & $1.3(0.8-2.1)$ \\
\hline \multicolumn{4}{|c|}{ Factor XII - Butter } \\
\hline QI & $73(29.1)$ & $63(25.2)$ & 1.0 \\
\hline QII & $57(22.7)$ & $62(24.8)$ & $0.8(0.5-1.3)$ \\
\hline QIII & 35 (13.9) & $62(24.8)$ & $0.5(0.3-0.8)$ \\
\hline QIV & $86(34.3)$ & 63 (25.2) & $1.2(0.7-1.9)$ \\
\hline \multicolumn{4}{|c|}{ Factor XIII - Bottled water } \\
\hline QI & $42(16.7)$ & $63(25.2)$ & 1.0 \\
\hline QII & 66 (26.3) & $62(24.8)$ & $1.6(1.0-2.7)$ \\
\hline QIII & $67(26.7)$ & 63 (25.2) & $1.6(1.0-2.7)$ \\
\hline QIV & 76 (30.3) & $62(24.8)$ & $1.9(1.1-3.1)$ \\
\hline
\end{tabular}

*BMI - body mass index.

†Quartiles of pack-years determined using former and current smokers combined: QI < 9.3; 9.3 $\leq$ QII $<22.2$; $22.2 \leq$ QIII $<34.7$; QIV $\geq 34.7$. $\ddagger$ Significant test for trend $(P \leq 0.05)$.

in the over-adjustment of a variable in the causal pathway $^{24}$. Similarly adjusting for BMI may have also resulted in an over-adjustment in the model. However, the estimates in the final logistic regression models did not change when BMI was not included in the model (data not shown). As BMI is a known risk factor of renal cell carcinoma, we chose to include it in our final models.

Although fruits in general tend to be associated with decreased risk, the vegetables also found to have this effect include Cruciferous and orange/green vegetables $^{3,5-7,10-12,23}$. A dietary pattern consisting mostly of fruits and vegetables was identified in both males and females in our data, and was inversely associated with disease, although not in the adjusted analysis. Furthermore, an increase in risk was associated with an 'unhealthy' diet in females, in which items such as broccoli and low-fat milk were infrequently consumed. In this factor, it was the absence of healthy foods that was associated with increased risk.
Despite reduced risk associated with 'fruits and vegetables' intake, we found an increased risk associated with a high intake of fruit juices in males. Maclure and Willett examined fruit juices as a risk factor but observed a non-significant decreased risk $^{1}$. Wolk et al. ${ }^{10}$ reported a decrease in risk with increasing intake levels of citrus fruit, while two studies did not observe an association between citrus fruits and renal cell carcinoma, except in nonsmokers $^{12,13}$.

Our data are consistent with an increased risk of fluid intake, per se. While Kreiger et $a{ }^{4}{ }^{4}$ did not report significantly increased risk associated with beverage intake, fluid intake has been shown to be positively associated with the risk of bladder cancer in some $e^{25-27}$ but not all ${ }^{28-30}$ studies.

One limitation of factor analysis is its subjectivity ${ }^{31}$. There are no accepted criteria for (1) the number of variables, (2) the number of factors, (3) the values of factor loadings to determine variables that contribute most to a 
Table 5 Adjusted ${ }^{\star}$ ORs and $95 \%$ Cls for renal cell carcinoma risk and dietary patterns in final logistic regression model by sex

\begin{tabular}{ll}
\hline Dietary factor & OR $(95 \% \mathrm{CI})$ \\
\hline Females & \\
Factor II - Desserts & 1.0 \\
QI & $0.6(0.3-1.0)$ \\
QII & $1.2(0.7-2.0)$ \\
QIII & $1.4(0.8-2.2)$ \\
QIV & \\
Factor VIII - Unhealthy & 1.0 \\
QI & $1.1(0.6-1.8)$ \\
QII & $2.1(1.3-3.4)$ \\
QIII & $1.4(0.8-2.4)$ \\
QIV & \\
Males & \\
Factor II - Desserts & 1.0 \\
QI & $2.1(1.1-4.0)$ \\
QII & $3.3(1.8-6.1)$ \\
QIII & $3.7(2.0-6.8)$ \\
QIV & \\
Factor V - Juices & 1.0 \\
QI & $0.8(0.5-1.5)$ \\
QII & $2.2(1.3-3.8)$ \\
QIII & $1.7(1.0-3.1)$ \\
QIV & \\
Factor VII - Beef & 1.0 \\
QI & $0.9(0.5-1.6)$ \\
QII & $1.8(1.0-3.1)$ \\
QIII & $1.0(0.6-1.7)$ \\
QIV &
\end{tabular}

${ }^{*}$ Adjusted for age, smoking status and body mass index.

factor, (4) the method of rotation and (5) the naming of factors $^{31}$. Furthermore, the generalisability of the results has not been fully explored. Dietary factors are driven by the study instrument used to measure diet intake and the sample from which they are derived. Our dietary factors were based on 69 food items that were presented in the food-frequency questionnaire; we are limited in generalising from these factors to the entire dietary intakes of our subjects. It is possible that the dietary factors explaining the most variation in dietary choices could be replicated in other populations, whereas factors accounting for less of the variability may not contribute to the dietary analysis of other populations ${ }^{30}$.

Our dietary factors accounted only for a maximum of 15 to $20 \%$ of the variance, similar to that observed in previous studies $^{30,32}$. To explain greater variance, additional dietary factors would be needed although these added factors might be more difficult to interpret, representing smaller amounts of variance and the increasingly heterogeneous 'patterns'.

Although the recall of diet is greatly influenced by current diet, studies have indicated that a more reliable estimate of the past diet is attained if subjects are questioned directly about their past diet rather than their current $^{24,33}$. The questionnaire asked directly about dietary intake two years prior to the date of interview. Although diet tends to have some consistency over time, our diet information may not reflect subjects' past diet allowing for appropriate exposure and latency periods $^{24}$. Similarly, our study may be subject to recall bias as cases and controls may have differed in their recall of past diet, with the possibility that cases or controls consistently under- or overreported their dietary intakes.

Factor analysis used for dietary assessment in nutritional epidemiology is advantageous because it identifies diet factors as they exist in a population. When consumed concurrently, correlated dietary factors may have interactive effects that could have positive or negative effects on health. Therefore, by representing diets as food factors, the multidimensional aspect of diet may be captured. Furthermore, patterns also consider the non-use of foods, as negatively correlated food items will appear within diet patterns.

Results from this study support an association between a diet characterised by high fat and protein foods and increased risk of renal cell carcinoma. An unexpected association between a fruit juice pattern and the risk of renal cell carcinoma was also observed among males, despite a reduced risk associated with a 'fruits and vegetables' dietary pattern. Further investigation is warranted to examine if fluid intake rather than intake of the specific constituents of fruit juices may be responsible for this finding.

Factor analysis has been used to generate diet factors in hope of understanding the aetiology of renal cell carcinoma in greater depth. By investigating dietary patterns and their associations with cancer risk, the biological mechanism between diet and cancer remains less clear. However, diet patterns identified for disease prevention may be important in making dietary recommendations for disease prevention.

\section{References}

1 Maclure M, Willett W. A case-control study of diet and risk of renal adenocarcinoma. Epidemiology 1990; 1: 430-40.

2 McLaughlin J, Mandel J, Blot W, et al. A population-based case-control study of renal cell carcinoma. J. Natl. Cancer Inst. 1984; 72: 275-84.

3 McLaughlin J, Gao Y, Gao R, et al. Risk factors for renal-cell cancer in Shanghai. China. Int. J. Cancer 1992; 52: 562-5.

4 Kreiger N, Marrett L, Dodds L, et al. Risk factors for renal cell carcinoma: results of a population-based case-control study. Cancer Causes Control 1993; 4: 101-10.

5 Negri E, La Vecchia C, Franceschi S, et al. Vegetable and fruit consumption and cancer risk. Int. J. Cancer 1991; 48: 350-4.

6 Talamini R, Baron A, Barra S, et al. A case-control study of risk factor for renal cell cancer in northern Italy. Cancer Causes Control 1990; 1: 125-31.

7 Fraser G, Phillips R, Beeson L. Hypertension, antihypertensive medication and risk of renal carcinoma in California Seventh-Day Adventists. Int. J. Epidemiol. 1990; 19: 832-8.

8 Armstrong B, Doll R. Environmental factors and cancer incidence and mortality in different countries, with special reference to dietary practices. Int. J. Cancer 1975; 15: $617-31$.

9 Wynder E, Mabuchi K, Whitmore W. Epidemiology of adenocarcinoma of the kidney. J. Natl. Cancer Inst. 1974; 53: $1619-34$. 
10 Wolk A, Gridley G, Niwa S, et al. International renal cell cancer study. VII. Role of diet. Int. J. Cancer 1996; 65: 67-73.

11 Wolk A, Lindblad P, Adami H. Nutrition and renal cell cancer. Cancer Causes Control 1996; 7: 5-18.

12 Lindblad P, Wolk A, Bergstrom R, et al. Diet and risk of renal cell cancer: a population-based case-control study. Cancer Epidemiol. Biomark. Prev. 1997; 6: 215-23.

13 Chow W, Gridley G, McLaughlin J, et al. Protein intake and risk of renal cell cancer. J. Natl. Cancer Inst. 1994; 86: 1131-9.

14 Johnson K, Mao Y, Argo J, et al. The National Enhanced Cancer Surveillance System: a case-control approach to environment-related cancer surveillance in Canada. Environmetrics 1998; 9: 495-504.

15 World Health Organization (WHO). International Classification of Diseases. Manual of the International Statistical Classification of Diseases, Injuries, and Causes of Death (Nineth Revision). Vol. 1. Geneva: WHO, 1977.

16 Villeneuve P, Johnson K, Kreiger N, et al. Risk factors for prostate cancer: results from the Canadian National Enhanced Cancer Surveillance System. Cancer Causes Control 1999; 10: 355-67.

17 SAS Institute, Inc., SAS Version 6.12. Cary, NC: SAS Institute, Inc., 1996.

18 Breslow N, Day N. Statistical Methods in Cancer Research. Vol. I. The Analysis of Case-Control Studies. IARC Scientific Publication No. 32. Lyons, France: International Agency for Research on Cancer (IARC), 1980; 5-338.

19 Statistics and Epidemiology Research Corporation and Cytel Software Corporation. EGRET ${ }^{\circledR}$. Seattle, WA: Statistics and Epidemiology Research Corporation and Cytel Software Corporation, 1993.

20 McCredie M, Ford J, Stewart J. Risk factors for cancer of the renal parenchyma. Int. J. Cancer 1988; 42: 13-6.

21 Prineas R, Folsom A, Zhang Z, et al. Nutrition and other risk factors for renal cell carcinoma in postmenopausal women. Epidemiology 1997; 8: 31-6.
22 Benichou J, Chow W, McLaughlin J, et al. Population attributable risk of renal cell cancer in Minnesota. Am. J. Epidemiol. 1998; 148: 424-30.

23 Mellemgaard A, McLaughlin J, Overvad K, et al. Dietary risk factors for renal cell carcinoma in Denmark. Eur. J. Cancer 1996; 32A: 673-82.

24 Willett W. Nutritional Epidemiology, 2nd ed. New York: Oxford University Press, 1990.

25 Vena J, Graham S, Freudenheim J, et al. Drinking water, fluid intake, and bladder cancer in western New York. Arch. Environ. Health 1993; 48: 191-8.

26 Claude J, Kunse E, Frentzel-Beyme R, et al. Life-style and occupational risk factors in cancer of the lower urinary tract. Am. J. Epidemiol. 1986; 124: 578-89.

27 Jensen OM, Wahrendorf J, Knudsen JB, et al. The Copenhagen case-control study of bladder cancer II. Effects of coffee and other beverages. Int. J. Cancer 1986; 37: $651-7$.

28 Michaud D, Spiegelman D, Clinton S, et al. Fluid intake and the risk of bladder cancer in men. N. Engl. J. Med. 1999; 340: 1390-7.

29 Pohlabeln H, Jocket K, Bolm-Audroff U. Non-occupational risk factors for cancer of the lower urinary tract in Germany. Eur. J. Epidemiol. 1999; 15: 411-9.

30 Slattery M, Boucher K, Caan B, et al. Eating patterns and risk of colon cancer. Am. J. Epidemiol. 1998; 148: 4-16.

31 Slattery M, Boucher K. The senior authors' response: factor analysis as a tool for evaluating eating patterns. Am. J. Epidemiol. 1998; 148: 20-1.

32 Randall E, Marshall J, Graham S, et al. Patterns in food use and their associations with nutrient intakes. Am.J. Clin. Nutr. 1990; 52: 739-45.

33 Byers T, Marshall J, Anthony E, et al. The reliability of dietary history from the distant past. Am. J. Epidemiol. 1987; 125: 999-1010. 